\title{
Browsing the Real World using Organic Electronics, Si-Chips, and a Human Touch.
}

Magnus Berggren, Daniel Simon, D Nilsson, P Dyreklev, P Norberg, S Nordlinder,

PA Ersman, G Gustafsson, Jacob Wikner, J Hederén, and Hentzell

\section{Linköping University Post Print}

\section{Tweet}

N.B.: When citing this work, cite the original article.

Original Publication:

Magnus Berggren, Daniel Simon, D Nilsson, P Dyreklev, P Norberg, S Nordlinder, PA Ersman, G Gustafsson, Jacob Wikner, J Hederén, and Hentzell, Browsing the Real World using Organic Electronics, Si-Chips, and a Human Touch., 2016, Advanced Materials, (28), 10, 1911-1916.

http://dx.doi.org/10.1002/adma.201504301

Copyright: Wiley-VCH Verlag

http://www.wiley-vch.de/publish/en/

Postprint available at: Linköping University Electronic Press

http://urn.kb.se/resolve?urn=urn:nbn:se:liu:diva-125994 


\title{
Browsing the Real World using Organic Electronics, Si-chips and a Human Touch
}

\author{
Magnus Berggren ${ }^{1}$, Daniel T. Simon ${ }^{1}$, David Nilsson ${ }^{2}$, Peter Dyreklev², Petronella \\ Norberg $^{2}$, Staffan Nordlinder ${ }^{2}$, Peter Andersson Ersman ${ }^{2}$, Göran Gustafsson ${ }^{2}$, J Jacob \\ Wikner ${ }^{3}$, Jan Hederén ${ }^{4}$ and Hans Hentzel/ ${ }^{5}$ \\ ${ }^{1}$ Laboratory of Organic Electronics, ITN, Linköping University, SE-601 74 Norrköping, Sweden \\ ${ }^{2}$ Acreo Swedish ICT, Box 787, SE-601 17 Norrköping, Sweden, \\ ${ }^{3}$ Department of electrical engineering, Linköping University, SE-581 83 Linköping, Sweden \\ ${ }^{4}$ DU Radio, Ericsson AB, SE-583 30 Linköping, Sweden \\ ${ }^{5}$ Swedish ICT Research, Box 1151, SE-164 26 Kista, Sweden
}

Organic electronics have been developed according to an orthodox doctrine advocating "allprinted", "all-organic" and "ultra-low-cost" primarily targeting various e-paper applications. In order to harvest from the great opportunities afforded with organic electronics potentially operating as communication and sensor outposts within existing and future complex communication infrastructures, high-quality computing and communication protocols must be integrated with the organic electronics. Here, we debate and scrutinize the twinning of the signal processing capability of traditional integrated silicon chips with organic electronics and sensors, and to use our body as a natural local network with our bare hand as the browser of the physical world. The resulting platform provides a body network, i.e. a personalized web, comprised of e-label sensors, bioelectronics and mobile devices that together make it possible to monitor and record both our ambience as well as health status parameters, supported by the ubiquitous mobile network and the resources of the "cloud". 


\section{Introduction}

Printed organic electronics, bioelectronics, silicon chip technology (Si-chips) and body area networks (BANs) are four building blocks that, when combined, offer a new powerful foundation that bridges the gap between signaling and communication of biology and the ambience, information technology and big data.

It is a fact that we, humans, are designed to touch things. During evolution our hands developed into a versatile multi-toolkit that serves us throughout basically all our daily duties. The combination of somatosensory recording, eyesight and proprioception of hand position makes our hands and fingers become our natural devices for haptic perception ${ }^{1}$. This helps us to generate illusions of surrounding objects, characterize and monitor our ambience in general and to assist us in basically all "hands-on" actions.

Printed organic electronics (Fig.1), integrated as e-labels ${ }^{2}$ including, e.g., sensors ${ }^{3}$ and identification codes, adhered onto objects in our ambience and on our body, promise distributed monitoring and recording of a vast array of chemical, physical and biological parameters. Dedicated printed sensors can measure the presence and concentration of various relevant compounds ${ }^{4,5}$. The sensor signals are then processed into data that is transmitted through an antenna or connector to devices for further data handling, and then possibly also transferred into the Internet cloud.

Organic electronic materials are soft and flexible, are compatible and stable when integrated with other systems, and desired combined chemical, electronic and ionic functionality can be defined within the materials themselves via chemical synthesis. These properties make organic electronics unique for a generic printed electronics platform. The same properties also provide unique advantages in an emerging organic bioelectronics platform serving as the bridge across the signaling gap between biology (ions, molecules) and technology (electrons). For instance, organic electronics have proven outstanding in translating electronic addressing signals into complex and precise delivery of biological (ionic, molecular) signals. Further, a wide range of different organic electronic sensors and electrodes are presently developed to monitor physiology. When integrated, these organic electronic delivery ${ }^{6}$ and sensor ${ }^{7}$ devices promise in vivo recording and auto-regulation of physiology, targeting future therapy and wellness monitoring (Fig. 1).

Si-chips, providing routing and processing of signals, are today pick-and-place-mounted on labels and flexible carriers at speeds up to, and even beyond, 20000 units-per-hour ${ }^{8}$. A range of different network and communication protocols have been developed for electronics during the last decades that extend connectivity and distribution of the web throughout our society. Body area networks (BANs), based on traditional Si-chip technology, have been proposed and developed ${ }^{9,10}$ and standards are also evolving ${ }^{11}$. These take use of the human body as a communication channel 
with, for instance, our hands as the galvanic or capacitive connectors. The BAN technology enables body-coupled communication (BCC) networks and is today being explored for applications such as monitoring patients' health status parameters ${ }^{12}$.

There are great opportunities for printed integrated (printegrated) systems and organic bioelectronics. Unfortunately, many of these opportunities are limited by the capabilities and poor stability of organic electronic materials and devices, in particular for applications where high-end signal processing and communication protocols are needed, such as in BAN and BCC applications. In comparison, higher-performance CMOS circuits are currently being explored and utilized for a variety of internet-of things and e-label technologies. To enable the promise of both low-cost and high performance for such ubiquitous electronics, "traditional" Si-based CMOS techniques will need to be augmented with other technologies, in particular printed electronics and especially organic electronics. We discuss on this silicon-organic merger and the great opportunities that arise as the two technologies complement each other.
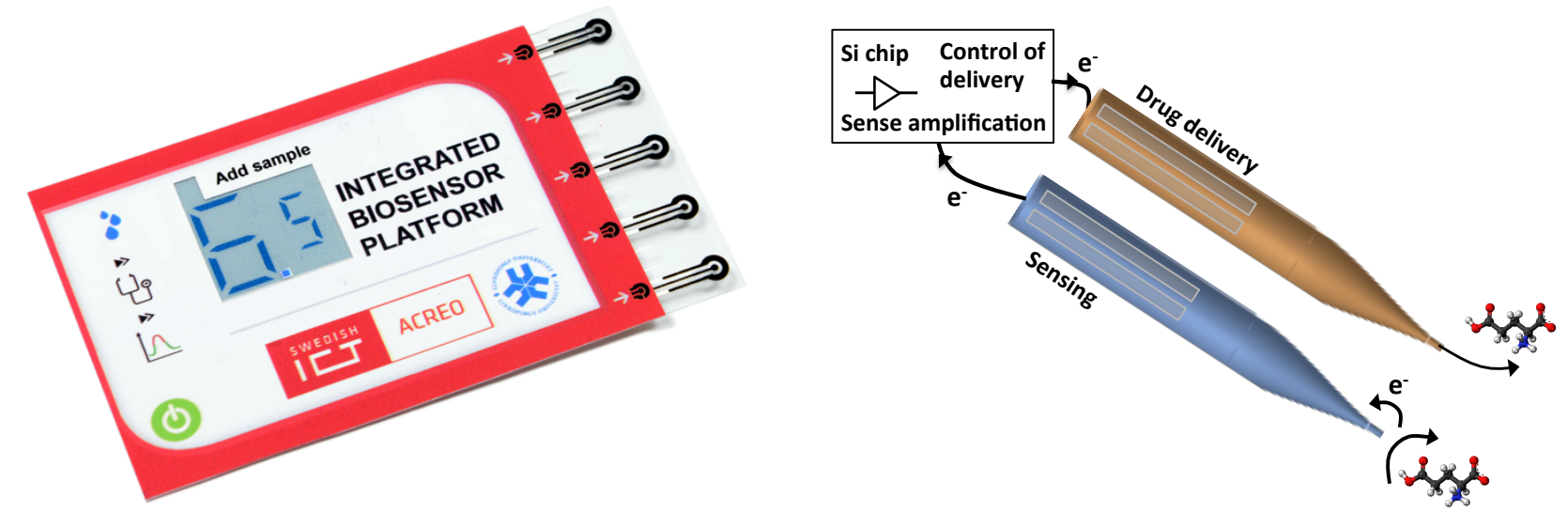

Figure 1. Left, a printegrated $e$-label for distributed sensing and diagnostics, comprising two separate Si-chips, screen-printed sensors, electrochromic displays elements, interconnects, a push-button and a printed battery. Right, organic electronic materials are today explored as a translator technology between electronics and biology, targeting medical therapy. Si chip technology can provide sense amplification and control of organic electronic sensors and drug delivery devices, respectively, in a feedback-regulated fashion (e-gland).

When printegrated systems and organic bioelectronics are combined with Si-chip technology many interesting opportunities arise. Here, we suggest one: Let us consider printegrated e-labels (on our body and in our ambience) and organic bioelectronics systems (implanted in our body), both combined with Si-chips for signal processing and connectivity. In the e-labels, various printegrated devices, i.e., sensors, capacitive or inductive antennas/electrodes, energy scavenger/conversion/ storage devices and circuitry, are combined with one or several embedded Si-chips. Various organic bioelectronics devices, i.e., sensors and delivery devices, are integrated into an electronic "gland" including Si-chips and antennas. Such an $e$-gland system enables feedback-regulated triggering and 
control over the release of pharmaceuticals. Then, if the case accessory of a mobile communication device includes an antenna/electrode, a BCC network is established that turns the body into your individual, local web. The hands become the browser of the world, grabbing sensory information from distributed $e$-labels on and around your body. This information is first processed locally in your mobile device and selected data can be transferred to the World-Wide-Web for further service and actions (Fig. 2). Finally, instructions from the cloud, relying on the stronger processing power outside the mobile device, will be relayed back into the $\mathrm{BCC}$ to regulate e.g. medication dispensed by the e-gland, in vivo.

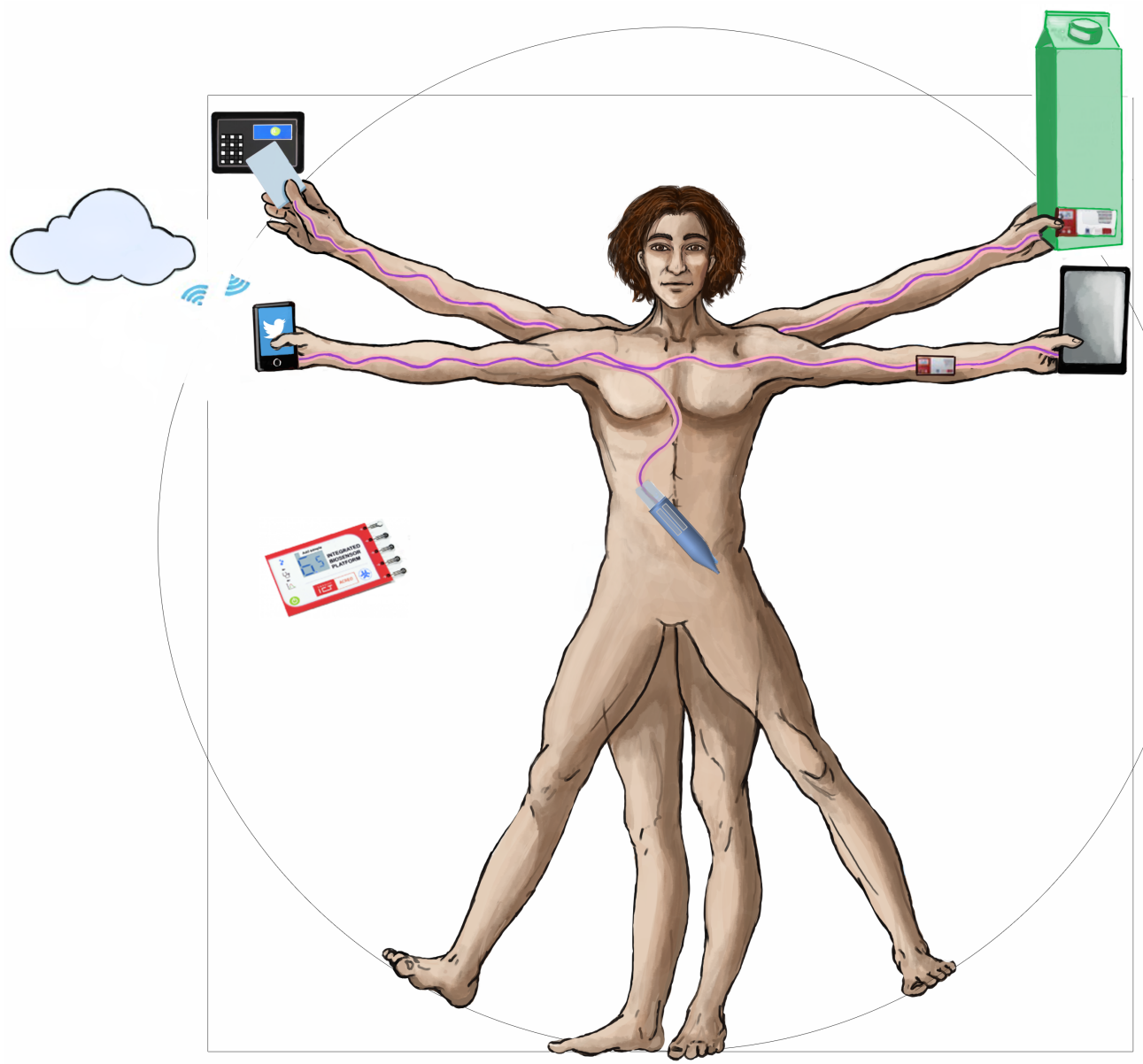

Figure 2. Your hands as the browser of the Real World Web; $e$-labels distributed around and on your body connected to your mobile devices utilizing your body as a personal network to regulate the dispensing of in vivo bioelectronics devices.

With the mobile phone in your pocket and printegrated e-labels adhered onto surfaces and items, one can simply walk around and browse things and surfaces to monitor a vast array of status parameters. In an extension, these $e$-labels can be further developed into skin patches and the BAN could also include communication with various e-glands. Together, a technology platform is 
derived that records and process physical, biological and chemical parameters of your ambience and your body. Selected data can be submitted to healthcare and wellness providers and, after further data processing and decision-making, your health status can be presented and automatic and remote medication can be initiated, in vitro and in vivo. In other words, the suggested technology enables remote diagnostics and therapy, a technology that could help to combat the rapidly increasing costs of health care in our society.

The proposed technology is successful only if it is based on the integration of (printed) organic electronics, bioelectronics and the highly competent Si-chips; a technology standard for this integration must be established soon in order to swiftly blaze the trail for the Internet of everything combined with BAN applications. In parallel, with the BAN technology systems and associated protocols at hand, $e$-labels and $e$-glands can define local personal webs at the same time as they serve as the outposts of a future highly expanded World-Wide-Web. This technology is highly sought-after - it can make significant impact on several areas of our society, also beyond health care, e.g., environmental monitoring, food safety and individual security.

To explore the feasibility and to identify scientific and engineering challenges of the RealWorld-Web platform, we developed two different $e$-labels. First, we produced a printed biosensor $e$ label comprising Si-chips with an array of different printegrated devices. Secondly, we fabricated an $e$-label to explore the feasibility to transfer data, through your body, between a mobile device and different distributed e-labels, adhered onto your body or onto dedicated devices and surfaces of your ambience.

\section{Printegrated electronics hand-in-hand with Si-chips on e-labels - integration challenges and opportunities}

The first e-label, see Fig. 1, represents a printegrated biosensor prototype. This first $e$-label includes several all-printed devices: a biosensor, interconnects, a push-button and electrochromic 7segmented displays, and it also hosts two Si-chips. The entire $e$-label is powered with a printed battery, which is manufactured separately and self-adhesively integrated with the $e$-label as the final manufacturing step. All printegrated devices are made via screen-printing and the two Si-chips are pick-and-place mounted using standard production and assembly techniques. When used, the $e$ label is turned on and a sample is then added onto the sensor area. The display provides simple instructions and updated information to the user. All data handling, electrical probing and analysis of the sensor is performed by the Si-chips and the sensing data is finally shown in the printed display. The second $e$-label exemplifies an ID-tag for BAN communication applications, which in part is manufactured and integrated in the same way as the first $e$-label, but with another choice of Si-chips and capacitive antennas. Our forecasted production cost per $e$-label unit indeed justifies 
disposable sensors and diagnostic strips for single-use applications. But, integration and task distribution between printegrated devices and the Si-chips must be carefully balanced.

The silicon chips, here utilized in our $e$-labels, include analogue and digital circuitry to receive and handle sensory input, to perform signal processing and to transmit information to antennas and displays. The total circuit area of each silicon chip is less than $1 \mathrm{~mm}^{2}$. As the $e$-label becomes more versatile and complex it will include more and more printegrated devices such as a set of dedicated sensors, extended display functionality, more interfaces, etc. This will typically not impact the circuit area of the Si-chip to any large extent. However, the number of contact pads scales proportionally with the number of I/Os necessary to exclusively communicate with all the printegrated devices; in fact the number of pads can easily exceed 20 even for a relatively simple $e$ label system. In that case it turns out that the pads will occupy most the area of the Si-chip, thus making the technology expensive, in fact too expensive for many of the suggested e-label applications. In addition, the assembly of these chips is relatively much more expensive than $\mathrm{Si}$ chips with only a few contact pads. First, printegrated transistor circuits, in the form of shift registers, de-multiplexers and multiplexers, can assist as a switchboard for Si-chips, thus allowing us to considerably reduce the number of pads, see Fig. 3. With this strategy, as few as perhaps four pads and a common ground plane, on the Si-chip can orchestrate a wide set of individual printegrated devices hosted on the $e$-label. Secondly, most organic electronic devices and circuits suffer from various invariances, hysteresis and/or stress effects that often relate to a large variation in the charge transport characteristics vs. bias, humidity and temperature. In Si-chips, temperature sensing can easily be defined together with proper circuits that can actively compensate for instability of the organic electronics. Thirdly, printegration for BAN applications and integration with the Si-chips also requires several traditional passive components, e.g. resistors, capacitors, as well as inductors with high Q values and low leakage. Fourthly, it should also be possible to print non-volatile read-and-write memories to further assist the Si-technology to keep it inexpensive. Finally, as used, the e-labels will be exposed to wear-and-tear, human touch, as well as significant electrostatic discharge (ESD) events. The $e$-labels are presently powered with batteries, but in many applications other energy sources will be possible and required for sustainability reasons. The emerging field of printed energy scavenging, harvesting and storage are certainly promising in this context. A combination of printed batteries, supercapacitors, energy scavengers and charge pumps in combination with printed balancing transistor circuits provides powering of the entire $e$-label during long-term storage and short-term usage, potentially requiring high power. 


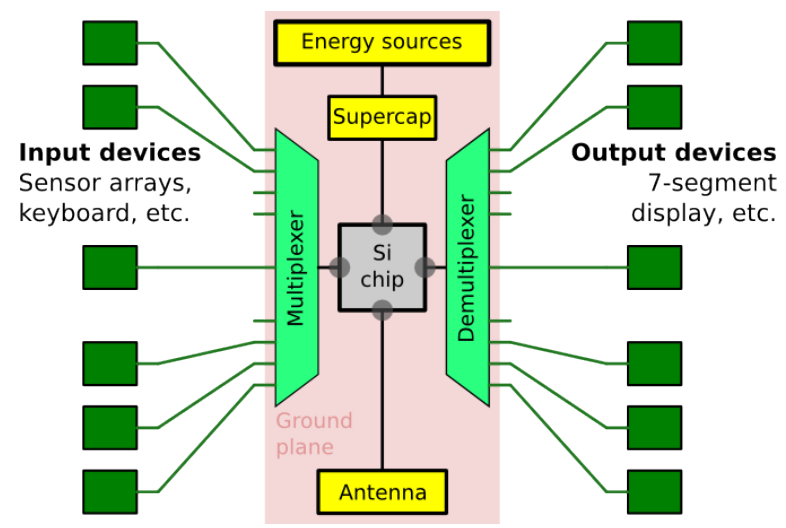

Figure 3. Illustration of the concept aiming to reduce the number of chip I/O pads using printed organic electronics (green) multiplexers and auxiliary logics.

\section{e-labels for BAN communication}

To use the power of touch we must obey the laws of physics and nature. All human beings are a network of capacitors and resistors. To utilize the body as a communication pathway we then need to seek for capacitive communication. The radio waves associated with modern wireless communication, in contrast, propagate poorly in the human body, since body fluids, e.g. aqueous electrolyte, represent 60 percent of our body volume.

In BAN applications using capacitive-coupled techniques, different protocols can be used to transfer data through the body dependent on the bit rate ${ }^{9,10,13}$. Using one of the high-speed communication protocols, we were able to transfer pictures from a smart phone to a monitor device with only our bare hands touching both devices simultaneously. In its extended version, and in the right environment, speeds up to $8 \mathrm{Mbps}$ were reached that allowed us to stream video through our body. In this proof-of-concept demonstrator, all devices were made from traditional electronic components.

A high-speed communication scenario in BAN application, however, is not necessarily what we are striving for in the present $e$-label target outlined in this commentary. Instead, in a low-data rate scenario, we wish to transfer just short messages, keys, sensor data, identity information and possibly also security codes, i.e. considerably less data as compared to streaming applications.

We have further demonstrated the use of a commercial provider's BCC development kit to implement compact labels based on printegrated systems with Si-chips mounted in and to them. In this scenario, we have embedded the $e$-labels in soup cans, parcels, and posters. Not only identification codes can be demonstrated. For example, we are able to actively interact and, for example, alert if the cans contain anything that could cause allergic reactions for the individual holding the can. This demonstration is outlined in Fig. 4. In this case, we have a can containing 
chocolate, but also nuts. When the user touches the can, the app, in the mobile device, also alerts the user indicating a possible allergen. This is specific to the user, who is allergic. In the extended form, we could also let the $e$-label itself sense and monitor a potentially hazardous or harmful parameter and not only rely on the programmed information based on the can ID. Further on, the $e$-label can also include sensor and diagnostic functionality related to healthcare and wellness. Security applications utilizing a "hand-shaking" between medical packages and the patient could even assure and guide for correct dosage.

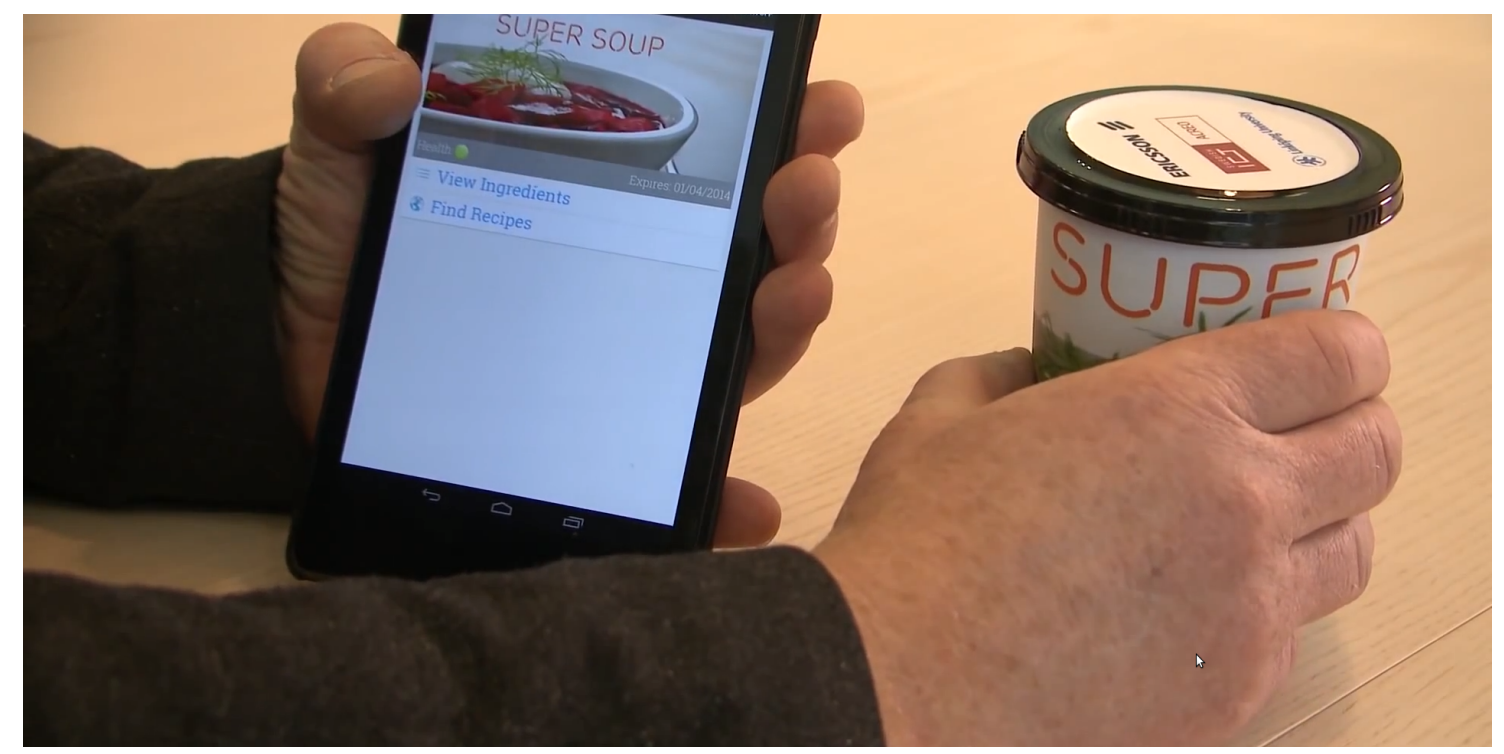

Figure 4. Body area network demonstration of an $e$-label (adhered on to a cup, left in the picture) communicating its identity, through the human body, to a tablet ${ }^{14,15}$.

As with most systems there are limitations associated with the capacitive-coupled technique. We still need to handle interference, eavesdropping, data loss, and cases were signal levels essentially are lost. For example, if objects are fully encapsulated in isolating materials, we must expose the capacitive antenna to enable a "human touch". There are more challenges that must be addressed, and there are many different user scenarios and channel characteristics. The human body behaves differently as a channel depending on, for example, height and girth. It also depends on the relative position of reader and tag; if the reader is stored in the back pocket or in the hand, or included in your wristband.

\section{Discussion and future outlook}

To bring organic electronics-Si-chip integration forward, we need to address a number of issues, in particular those related to development of organic electronic materials. Here we identify some of these key issues:

- The ideal silicon-organic balance: from a system and function point of view, what should be 
comprised of printed organic electronics and bioelectronics and what should be defined by Si-chip circuit systems? Basically, how should we partition the combined systems? One guideline to finding this balance could be selecting technologies that can extend across large areas of the e-paper (printed organic electronics) compared to high-performing communication and computing taken care of very locally (Si-chip).

- Which algorithms and protocols, defined in Si-chip technology, combined with temperature, humidity, or other environmental sensors, can best compensate for some of the degradations (e.g., bias stress, aging, and drift of performance) that printed organic electronics suffer from? For the effects cannot be compensated for let us then focus on development of materials and characteristics to solve these remaining deficiencies in the organic electronics, most likely those related to photo-oxidation affecting mechanical and electrical properties, compatibility, manufacturing, and flexibility.

- With shift registers and (de-)multiplexers, all defined in printed organic electronics, the number of contact pads can be reduced in the Si-chips. However, the signal compatibility between the Si-chip technology and organic electronics is still a great challenge. We need therefore to focus on the development of materials and device concepts that guarantee both proper stability and signal quality, in an overall technology that operates at voltage levels below $3 \mathrm{~V}$, such that the complexity of the system is kept at a minimum by avoiding e.g. charge pump circuitry.

- In many applications it is desired to combine solid-state electronic device systems (e.g. transistors, didoes, memories) with electrochemical ones (e.g. sensors, batteries, electrochromic displays). We suggest that one focus is directed towards development of compatible materials and devices so that these two classes of materials can co-operate within the same e-label. Another focus could perhaps be directed towards the development of highly conducting mixed ionic-electronic conductors (e.g. supercapacitors, batteries).

At one end of our body, we have the printegrated-Si-chip e-label and bioelectronics-Si-chip $e$ gland solution combined with a capacitive intra-connected BAN enabling its own protected natural communication network. At the other end we have the smart phone in the hand or pocket using technology and nature to create a game-changing platform. This novel platform makes it possible to browse and surf the Real-World-Web by the touch of the hand.

Real-World-Web browsing will make the already powerful World-Wide-Web radically much wider! 


\section{Acknowledgement}

The authors would like to thank Knut and Alice Wallenberg foundation, the Önnesjö Foundation, VINNOVA, The Swedish Foundation for Strategic Research for financial support. 


\section{References}

1 Amedi, A., Malach, R., Hendler, T., Peled, S. \& Zohary, E. Visuo-haptic object-related activation in the ventral visual pathway. Nature Neuroscience 4, 324-330 (2001).

2 Berggren, M., Nilsson, D. \& Robinson, N. D. Organic materials for printed electronics. Nature Materials 6, 3-5 (2007).

3 Noguchi, Y., Sekitani, T. \& Someya, T. Organic-transistor-based flexible pressure sensors using ink-jet-printed electrodes and gate dielectric layers. Applied Physics Letters 89, 253507-253511 (2006).

4 Briand, D., Oprea, A., Courbat, J. \& Bârsan, N. Making environmental sensors on plastic foil. Materials Today 14, 416-423 (2011).

5 Bernards, D. A. et al. Enzymatic sensing with organic electrochemical transistors. Journal of Materials Chemistry 18, 116-120 (2008).

6 Simon, D. T. et al. Organic electronics for precise delivery of neurotransmitters to modulate mammalian sensory function. Nature Materials 8, 742-746 (2009).

$7 \quad$ Khodagholy, D. et al. In vivo recordings of brain activity using organic transistors. Nature Communications 4 (2013).

8 Koepp, R., Allen, T., Fassett, J. \& Teng, A. in Proceedings of the IEEE/CPMT International Electronics Manufacturing Technology (IEMT) Symposium.

9 Zimmerman, T. G. Personal area networks: Near-field intrabody communication. IBM Systems Journal 35, 609-617 (1996).

10 Wegmueller, M. et al. Measurement system for the characterization of the human body as a communication channel at low frequency. Annual International Conference of the IEEE Engineering in Medicine and Biology - Proceedings 7, 3502-3505 (2005).

11 Attard, S. \& Zammit, S. An empirical investigation of the capacitive Body Coupled Communications channel for Body Area Networks. RWW 2012 - Proceedings: 2012 IEEE Topical Conference on Biomedical Wireless Technologies, Networks, and Sensing Systems, BioWireleSS 2012, 85-88 (2012).

12 Zhou, J., Guo, A., Xu, J. \& Su, S. An optimal fuzzy control medium access in wireless body area networks. Neurocomputing 142, 107-114 (2014).

13 Song, S.-J., Cho, N., Kim, S., Yoo, J. \& Yoo, H.-J. A 2Mb/s Wideband Pulse Transceiver with Direct-Coupled Interface for Human Body Communications. Solid-State Circuits Conference, 2006. ISSCC 2006. Digest of Technical Papers. IEEE International 2278-2287, doi:10.1109/ISSCC.2006.1696290 (2006).

142014 , E. A. C. m. (http://www.ericsson.com/thinkingahead/visionaryideas/connected-me).

15 2014, E. A. C. p. (http://www.ericsson.com/thinkingahead/visionary-ideas/connectedprint). 